\title{
Estimation of Population Variance in Simple Random Sampling using Auxiliary Information
}

\author{
Sumaira Ajmal Khan \\ Lahore Garrison University, \\ Phase 6, DHA Lahore
}

\author{
Mehwish Nawaz \\ Lahore Garrison University, \\ Phase 6, DHA Lahore
}

\author{
Kushbkht M. Din \\ Lahore Garrison University, \\ Phase 6, DHA Lahore
}

\begin{abstract}
In this paper we propose a new estimator for the population variance using auxiliary information in simple random sampling. we derived a bias and mean square error equation of proposed estimator and compare with the bias and MSE of existing estimator and show that proposed estimator is more efficient than the existing estimators suggested by different authors such that Kadilar and Cingi (2005) [6] , Isaki(1983) [5]. We support this theoretical result with the help of a numerical illustration.
\end{abstract}

\section{Keywords}

Variance estimator, bias, MSE, simple random sampling, auxiliary information, Efficiency

\section{INTRODUCTION}

Auxiliary information has been used extensively in estimation of parameters like mean and variance since several decades.

Isaki (1983) [5] got inspiration from ratio estimator of finite population mean and proposed a ratio estimator, usually called classical estimator, of finite population variance. But Singh and Solanki (2013a) [12] claimed that Isaki (1983) [5] ratio estimator is the member of the class of estimators developed by Das and Tripathi (1978) [3]. Arcos and Rueda (1997) [2] suggested multivariate ratio estimator for population variance. Ahmed et al. (2000) [1] criticized the claim of Arcos and Rueda (1997) [2] , Kadilar and Cingi (2006a) [7] developed an estimator, ratio-type estimator of the mean of population, Kadilar and Cingi (2006b) [8] extended the idea of Isaki (1983) [5] ratio estimator for population variance . . Kadilar and Cingi (2006b) [8] involved the information available about coefficient of variation and coefficient of kurtosis of the auxiliary variable to generate these estimators under simple random sampling as well . Gupta and Shabbir (2008) [4] gave a hybrid class of variance estimators of population mean . Subramani and Kumarapandiyan (2012a) [9] modified the usual ratio-type estimator of Kadilar and Cingi (2006b) [8] for population variance using population median obtained from auxiliary variable . Subramani and Kumarapandiyan (2012b) [10] further modified the usual ratio-type variance estimators using lower and upper quartiles, inter-quartile range, quartile deviation and quartile average of the auxiliary variable . Subramani and Kumarapandiyan (2013) [11] developed another more efficient modified ratio-type estimator using median and coefficient of variation of the auxiliary variable.

\section{NOTATIONS}

$\mathrm{N}$ Population size $\mathrm{n}$ sample size

$\bar{Y}=\frac{\sum_{i=1}^{N} y_{i}}{N} \quad$ population mean of the study variable $\mathrm{y}$

$\bar{X}=\frac{\sum_{i=1}^{N} x_{i}}{N} \quad$ population mean of the auxiliary variable $\mathrm{x}$

$$
\begin{aligned}
& \bar{y}=\frac{\sum_{i=1}^{n} y_{i}}{n} \quad \text { sample mean of the study variable } \mathrm{y} \\
& \bar{x}=\frac{\sum_{i=1}^{n} x_{i}}{n} \quad \text { sample mean of the auxiliary variable } \mathrm{x} \\
& S_{y}^{2}=\frac{\sum_{i=1}^{N}\left(y_{i}-\bar{Y}\right)^{2}}{N-1} \quad \text { Population variance of the study variable } \\
& S_{x}^{2}=\frac{\sum_{i=1}^{N}\left(x_{i}-\bar{X}\right)^{2}}{N-1} \quad \text { Population variance of the auxiliary } \\
& \text { variable. } \\
& s_{y}^{2}=\frac{\sum_{i=1}^{N}\left(y_{i}-\bar{y}\right)^{2}}{n-1} \quad \text { sample variance of the study variable } \\
& S_{x}^{2}=\frac{\sum_{i=1}^{N}\left(x_{i}-\bar{x}\right)^{2}}{n-1} \quad \text { sample variance of the auxiliary variable } \\
& \lambda^{\prime}=\frac{1}{n} \quad C_{x}, C_{y}=\text { Coefficient of variations } \\
& \beta_{1(x)}=\frac{\mu_{03}^{2}}{\mu_{02}^{2}} \text { Skewness of the auxiliary variable } \\
& \beta_{2(\mathrm{x})}=\frac{\mu_{04}^{2}}{\mu_{02}^{2}} \text { Kurttosis of the auxuliary variable } \\
& \beta_{2(\mathrm{y})} \\
& =\frac{\mu_{40}^{2}}{\mu_{20}^{2}} \text { Kurtosis of the study variable } \\
& \mathrm{E}\left(e_{0}\right)=0 \quad, \quad \mathrm{E}\left(e_{1}\right)=0
\end{aligned}
$$

\section{EXISTING ESTIMATORS IN SIMPLE RANDOM SAMPLING}

\subsection{Isaki (1983) [5]}

Motivated by the estimator of the population mean $\hat{\mu}_{y R}=$ $\frac{\bar{y}}{\bar{x}} \bar{X}$ Isaki [5] suggested the ratio estimator of the variance of population using auxiliary information .

$t_{\text {Isaki }}=s_{y}^{2} \frac{s_{x}^{2}}{s_{x}^{2}}$

The MSE of the above estimator using first order approximation is

$$
\begin{aligned}
& \operatorname{MSE}\left(t_{\text {Isaki }}\right) \cong \lambda^{\prime} S_{y}^{4}\left\{\beta_{2}(y)+\beta_{2}(x)-2 h\right\} \\
& \operatorname{Or} \\
& \operatorname{MSE}\left(t_{\text {Isaki }}\right) \cong \lambda^{\prime} S_{y}^{4}\left\{\beta_{2}^{\prime}(y)+\beta_{2}^{\prime}(x)-2 h^{\prime}\right\} \\
& \operatorname{The~bias~is~} \\
& \operatorname{Bias}\left(t_{\text {Isaki }}\right) \cong \lambda^{\prime} S_{y}^{2}\left\{\beta_{2}(x)-h\right\} \\
& \operatorname{Or} \\
& \operatorname{Bias}\left(t_{\text {Isaki }}\right) \cong \lambda^{\prime} S_{y}^{2}\left\{\beta_{2}^{\prime}(x)-h^{\prime}\right\}
\end{aligned}
$$$$
\text { 3.2 Kadilar and Cingi (2005) [6] }
$$

Kadilar and Cingi [6] proposed the following class of estimators for population variance.

$t_{C H 1}=s_{y}^{2}\left[s_{x}^{2}-\beta_{2}(x)\right]^{-1}\left[S_{x}^{2}-\beta_{2}(x)\right]$ 


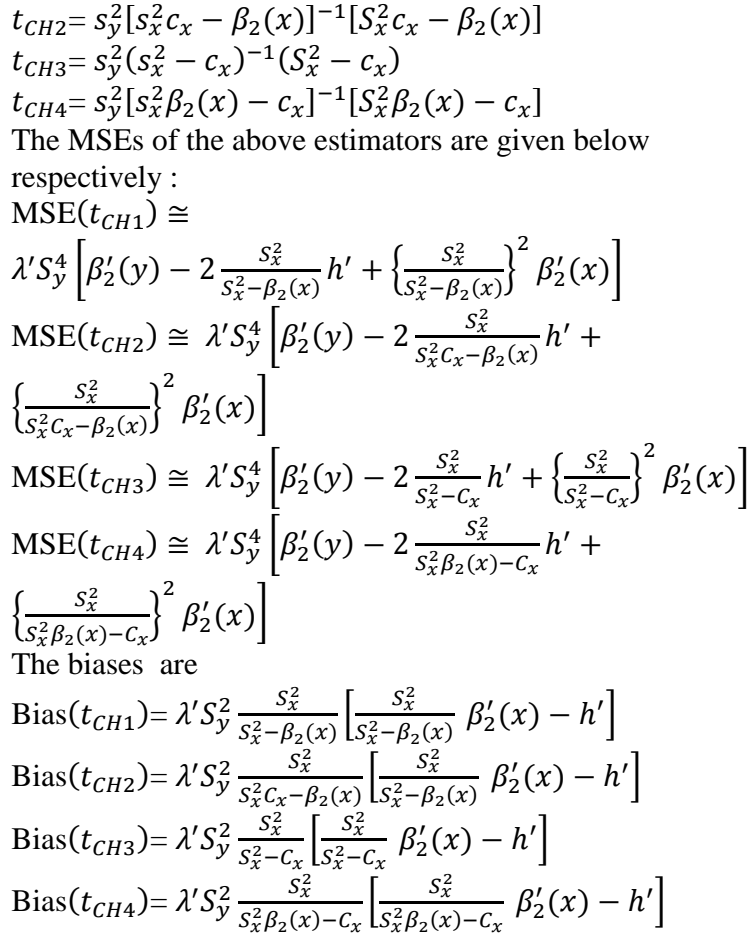

\subsection{Subramani and Kumarapandiyan}

\section{(2012b) [10]}

Subramani and Kumarapandiyan [10] suggested the following efficient estimator

Using quartiles and functions of quartiles of the auxiliary variable.

$$
\begin{aligned}
& t_{j g 1}=s_{y}^{2}\left(s_{x}^{2}+Q_{1}\right)^{-1}\left(s_{x}^{2}+Q_{1}\right) \\
& t_{j g 2}=s_{y}^{2}\left(s_{x}^{2}+Q_{3}\right)^{-1}\left(s_{x}^{2}+Q_{3}\right) \\
& t_{j g 3}=s_{y}^{2}\left(s_{x}^{2}+Q_{R}\right)^{-1}\left(s_{x}^{2}+Q_{R}\right) \\
& t_{j g 4}=s_{y}^{2}\left(s_{x}^{2}+Q_{D}\right)^{-1}\left(s_{x}^{2}+Q_{D}\right) \\
& t_{j g 5}=s_{y}^{2}\left(s_{x}^{2}+Q_{A}\right)^{-1}\left(s_{x}^{2}+Q_{A}\right) \\
& \text { Where } \\
& Q_{R}=Q_{3}-Q_{1} \\
& Q_{D}=\frac{Q_{3}-Q_{1}}{2} \quad Q_{A}=\frac{Q_{3}+Q_{1}}{2}
\end{aligned}
$$

And their respective mean squared errors are:

$$
\begin{aligned}
& \operatorname{MSE}\left(t_{j g 1}\right)=\lambda^{\prime} s_{y}^{4}\left[\beta_{2}^{\prime}(y)-2 \frac{s_{x}^{2}}{s_{x}^{2}+Q_{1}} h^{\prime}+\left\{\frac{s_{x}^{2}}{s_{x}^{2}+Q_{1}}\right\}^{2} \beta_{2}^{\prime}(x)\right] \\
& \operatorname{MSE}\left(t_{j g 2}\right)=\lambda^{\prime} s_{y}^{4}\left[\beta_{2}^{\prime}(y)-2 \frac{s_{x}^{2}}{s_{x}^{2}+Q_{3}} h^{\prime}+\left\{\frac{s_{x}^{2}}{s_{x}^{2}+Q_{3}}\right\}^{2} \beta_{2}^{\prime}(x)\right] \\
& \operatorname{MSE}\left(t_{j g 3}\right)=\lambda^{\prime} s_{y}^{4}\left[\beta_{2}^{\prime}(y)-2 \frac{s_{x}^{2}}{s_{x}^{2}+Q_{R}} h^{\prime}+\left\{\frac{s_{x}^{2}}{s_{x}^{2}+Q_{R}}\right\}^{2} \beta_{2}^{\prime}(x)\right] \\
& \operatorname{MSE}\left(t_{j g 4}\right)=\lambda^{\prime} s_{y}^{4}\left[\beta_{2}^{\prime}(y)-2 \frac{s_{x}^{2}}{s_{x}^{2}+Q_{D}} h^{\prime}+\left\{\frac{s_{x}^{2}}{s_{x}^{2}+Q_{D}}\right\}^{2} \beta_{2}^{\prime}(x)\right] \\
& \operatorname{MSE}\left(t_{j g 5}\right)=\lambda^{\prime} s_{y}^{4}\left[\beta_{2}^{\prime}(y)-2 \frac{s_{x}^{2}}{s_{x}^{2}+Q_{A}} h^{\prime}+\left\{\frac{s_{x}^{2}}{s_{x}^{2}+Q_{A}}\right\}^{2} \beta_{2}^{\prime}(x)\right]
\end{aligned}
$$

And the respective biases are

$$
\begin{aligned}
& \mathrm{B}\left(t_{j g 1}\right)=\lambda^{\prime} s_{y}^{2} \frac{s_{x}^{2}}{s_{x}^{2}+Q_{1}}\left[\frac{s_{x}^{2}}{s_{x}^{2}+Q_{1}} \beta_{2}^{\prime}(x)-h^{\prime}\right] \\
& \mathrm{B}\left(t_{j g 2}\right)=\lambda^{\prime} s_{y}^{2} \frac{s_{x}^{2}}{s_{x}^{2}+Q_{3}}\left[\frac{s_{x}^{2}}{s_{x}^{2}+Q_{3}} \beta_{2}^{\prime}(x)-h^{\prime}\right] \\
& \mathrm{B}\left(t_{j g 3}\right)=\lambda^{\prime} s_{y}^{2} \frac{s_{x}^{2}}{s_{x}^{2}+Q_{R}}\left[\frac{s_{x}^{2}}{s_{x}^{2}+Q_{R}} \beta_{2}^{\prime}(x)-h^{\prime}\right] \\
& \mathrm{B}\left(t_{j g 4}\right)=\lambda^{\prime} s_{y}^{2} \frac{s_{x}^{2}}{s_{x}^{2}+Q_{D}}\left[\frac{s_{x}^{2}}{s_{x}^{2}+Q_{D}} \beta_{2}^{\prime}(x)-h^{\prime}\right] \\
& \mathrm{B}\left(t_{j g 5}\right)=\lambda^{\prime} s_{y}^{2} \frac{s_{x}^{2}}{s_{x}^{2}+Q_{A}}\left[\frac{s_{x}^{2}}{s_{x}^{2}+Q_{A}} \beta_{2}^{\prime}(x)-h^{\prime}\right]
\end{aligned}
$$

\section{PROPOSED ESTIMATOR}

We proposed a new modified ratio type variance estimator of the auxiliary variable by using known value of kurtosis of the auxiliary variable. The modified ratio type variance estimator for population variance is defined as

$t_{\text {new }}=s_{y}^{2} \frac{S_{x}^{2}+S_{x} \beta_{2}(x)}{s_{x}^{2}+S_{x} \beta_{2}(x)}$

\subsection{Bias of Proposed Estimator}

$$
\begin{gathered}
t_{\text {new }}=S_{y}^{2}\left(1+e_{0}\right) \frac{S_{x}^{2}+S_{x} \boldsymbol{\beta}_{2}(x)}{S_{x}^{2}\left(1+e_{1}\right)+S_{x} \boldsymbol{\beta}_{2}(x)} \\
t_{n e w}=S_{y}^{2}\left(1+e_{0}\right)\left\{1+\frac{S_{x}^{2} e_{1}}{S_{x}^{2}+S_{x} \beta_{2}(x)}\right\}^{-1} \\
t_{\text {new }}=S_{y}^{2}\left(1+e_{0}\right)\left\{1+\Omega e_{1}\right\}^{-1}
\end{gathered}
$$

Where

$$
\Omega=\frac{S_{x}^{2}}{S_{x}^{2}+S_{x} \beta_{2}(x)}
$$

Expending the expression by using Taylor's series

$$
\begin{gathered}
\left.\left(\Omega e_{1}\right)^{3}+\cdots\right\} \\
t_{\text {new }}=S_{y}^{2}\left(1+e_{0}\right)\left\{1-\Omega e_{1}+\left(\Omega e_{1}\right)^{2}\right\} \\
t_{\text {new }}-S_{y}^{2}=S_{y}^{2}\left\{e_{0}-\Omega e_{1}+\left(\Omega e_{1}\right)^{2}-\Omega e_{0} e_{1}\right\} \\
\mathrm{E}\left(t_{\text {new }}-S_{y}^{2}\right)=\mathrm{E}\left\{S_{y}^{2}\left(e_{0}-\Omega e_{1}+\left(\Omega e_{1}\right)^{2}-\Omega e_{0} e_{1}\right)\right\} \\
\mathrm{B}\left(t_{\text {new }}\right)=\Omega \lambda^{\prime} S_{y}^{2}\left\{\Omega \beta_{2(x)}^{\prime}-h^{\prime}\right\}
\end{gathered}
$$$$
t_{\text {new }}=S_{y}^{2}\left(1+e_{0}\right)\left\{1-\Omega e_{1}+\left(\Omega e_{1}\right)^{2}-\right.
$$

\subsection{Mean Square Error of the proposed}

\section{Estimator}

Now comes MSE of the estimator

$$
\begin{aligned}
\operatorname{MSE}\left(t_{n e w}\right) & =\mathrm{E}\left\{S_{y}^{2}\left(e_{0}-\Omega e_{1}\right)\right\}^{2} \quad \text { ignore higher order } \\
& =S_{y}^{4}\left\{E\left(e_{0}^{2}\right)+\Omega^{2} E\left(e_{1}^{2}\right)-2 \Omega E\left(e_{0} e_{1}\right)\right\} \\
& =S_{y}^{4}\left\{\lambda^{\prime} \beta_{2(y)}^{\prime}+\Omega^{2} \lambda^{\prime} \beta_{2(x)}^{\prime}-2 \Omega \lambda^{\prime} h^{\prime}\right\} \\
& =\lambda^{\prime} S_{y}^{4}\left\{\beta_{2(y)}^{\prime}+\beta_{2(x)}^{\prime}\left(\Omega^{2}-2 \Omega \frac{h^{\prime}}{\beta_{2(x)}^{\prime}}\right)\right\} \\
& =\lambda^{\prime} S_{y}^{4}\left\{\beta_{2(y)}^{\prime}+\beta_{2(x)}^{\prime}\left(\Omega^{2}-\right.\right. \\
\left.\left.2 \Omega v^{\prime}\right)\right\} \ldots \ldots \ldots \ldots \ldots \ldots \ldots \ldots . . .(\mathrm{i}) &
\end{aligned}
$$

Where

$$
\therefore \quad v^{\prime}=\frac{h^{\prime}}{\beta_{2(x)}^{\prime}}
$$

In order to minimize MSE to Differentiate (i) partially w.r.t $\Omega$ and equating to zero

$$
\begin{aligned}
& \frac{\partial}{\partial \Omega} \operatorname{MSE}\left(t_{\text {new } 1}\right)=0 \\
& \lambda^{\prime} S_{y}^{4} \frac{\partial}{\partial \Omega}\left\{\beta_{2(y)}^{\prime}+\beta_{2(x)}^{\prime}\left(\Omega^{2}-2 \Omega v^{\prime}\right)\right\}=0 \\
& \Omega=v^{\prime}
\end{aligned}
$$




$$
\begin{aligned}
= & \lambda^{\prime} \boldsymbol{S}_{y}^{\mathbf{4}}\left\{\boldsymbol{\beta}_{2(\boldsymbol{y})}^{\prime}-\boldsymbol{\beta}_{2(x)}^{\prime} \boldsymbol{v}^{\prime 2}\right\} \\
= & \lambda^{\prime} \boldsymbol{S}_{y}^{\mathbf{4}} \boldsymbol{\beta}_{2}^{\prime}(\boldsymbol{y})\left\{\mathbf{1}-\boldsymbol{v}^{\prime \mathbf{2}} \frac{\boldsymbol{\beta}_{2(x)}^{\prime}}{\boldsymbol{\beta}_{2(y)}^{\prime}}\right\} \\
= & \lambda^{\prime} S_{y}^{4} \beta_{2(y)}^{\prime}\left\{1-\left(\frac{h^{\prime}}{\sqrt{\beta_{2(x)}^{\prime} \beta_{2(y)}^{\prime}}}\right)^{2}\right\}
\end{aligned}
$$

$\operatorname{MSE}\left(t_{\text {new }}\right)=\lambda^{\prime} S_{y}^{4} \beta_{2(y)}^{\prime}\left\{1-\left(\rho^{\prime}\right)^{2}\right\}$

Where

$$
\rho^{\prime}=\frac{h^{\prime}}{\sqrt{\beta_{2(x)}^{\prime} \beta_{2(y)}^{\prime}}}
$$

\section{PROPOSED ESTIMATOR}

To demonstrate the performance of the proposed estimator empirically in comparison to other estimators. I have used five data sets. The description of data sets is given below.

\subsection{Data 1}

Source: Gupta and Shabbir (2008) [4]

The available statistics are based on the data obtained from 104 villages existing in East Anatolia (Turkey) in 1999. $\mathrm{Y}$, the variable of interest represent apple 's production level per 100 tons

$\mathrm{X}$, the auxiliary variable shows number of apple trees (in100s).

\subsection{Data 2}

Source : Gupta and Shabbir (2008) [4]

The data collected from 278 villages/Towns/wards under the control of Gajole polstation (Malda district, west Bengal) India.

$\mathrm{Y}$, indicates number of agricultural labourers for the year 1971

$X$, the number of agricultural labourers for the year 1961

\subsection{Data 3}

Source : Rohini et al (Jan 2012)

Data collected from 142 Indian cities with population 0.1 million and above.

\begin{tabular}{|c|c|c|c|c|c|c|}
\hline \multicolumn{2}{|l|}{ Estimators } & \multirow{2}{*}{$\begin{array}{l}\text { Data 1 } \\
21.23184951\end{array}$} & \multirow{2}{*}{$\begin{array}{l}\text { Data2 } \\
1282.9966\end{array}$} & \multirow{2}{*}{$\begin{array}{l}\text { Data3 } \\
7900281.139\end{array}$} & \multirow{2}{*}{$\begin{array}{l}\text { Data4 } \\
12569.0763\end{array}$} & \multirow{2}{*}{$\begin{array}{l}\text { Dat5 } \\
224.623916\end{array}$} \\
\hline Isaki (1983) & $t_{\text {Isaki }}$ & & & & & \\
\hline \multirow[t]{4}{*}{ Kadilar \& Cingi (2005) } & $t_{C H 1}$ & 21.23185393 & 1413.1212 & 7900391.028 & 12569.802 & 225.768988 \\
\hline & $t_{C H 2}$ & 21.231852 & 1342.2774 & 7900326.9 & 12569.346 & 225.12942 \\
\hline & $t_{T C H 3}$ & 21.23184993 & 21.231843 & 21.23184993 & 21.2318499 & 21.2318499 \\
\hline & $t_{C H 4}$ & 21.231853 & 21.231853 & 21.231853 & 21.231853 & 21.231853 \\
\hline \multirow{5}{*}{$\begin{array}{l}\text { Subramani \& } \\
\text { Kumarapandiyan } \\
(2012 b)\end{array}$} & $t_{j g 1}$ & 21.23184289 & 1074.8284 & 7900199.561 & 12568.417 & 222.857906 \\
\hline & $t_{j g 2}$ & 21.23182966 & 737.70151 & 7900036.406 & 12567.0984 & 219.349828 \\
\hline & $t_{j g 3}$ & 21.23183627 & 894.52749 & 7900117.983 & 12567.7577 & 221.099891 \\
\hline & $t_{j g 4}$ & 21.23184289 & 1074.8284 & 7900199.561 & 12568.417 & 222.857906 \\
\hline & $t_{j g 5}$ & 21.23183627 & 894.52749 & 7900117.983 & 12567.7577 & 221.099891 \\
\hline Proposed Estimator & $t_{\text {new }}$ & 21.13030158 & -350.02315 & 7210041.419 & 11436.3159 & 58.1996276 \\
\hline
\end{tabular}

Y: 1971 population census

$\mathrm{X}: 1961$ population census

Table.1 Bias of the Existing and the Proposed Estimator $\mathrm{n}=40$

\subsection{Data 4}

Source: Kadilar and Cingi (2005) [6] ratio estimators for the population variance in simple random sampling.

The data obtained from 94 villages in Mediterranean(Turkey) in 1999.

Y: the variable of interest represents apple's production level per 100 tons

$\mathrm{X}$ : the auxiliary variable shows number of apple trees(in $100 \mathrm{sec}$ ).

\subsection{Data 5}

Source: Kadilar and Cingi (2005) [6] ratio estimators for the population variance in simple random sampling.

The available statistics obtained from 173 villages existing In East and southeast

Y: the variable of interest represents apple's production level

\begin{tabular}{|c|c|c|c|c|c|}
\hline & Data 1 & Data 2 & Data 3 & $\begin{array}{l}\text { Data } \\
4\end{array}$ & $\begin{array}{l}\text { Dat } \\
\text { a } 5\end{array}$ \\
\hline $\mathrm{N}$ & 104 & 278 & 142 & 94 & 173 \\
\hline $\mathrm{n}$ & 20 & 30 & 40 & 38 & 2 \\
\hline $\bar{Y}$ & 6.254 & 39.068 & $\begin{array}{l}4015.21 \\
83\end{array}$ & $\begin{array}{l}93.8 \\
4\end{array}$ & 4.04 \\
\hline $\bar{X}$ & $\begin{array}{l}13931 . \\
683\end{array}$ & 25.111 & $\begin{array}{l}2900.38 \\
72\end{array}$ & $\begin{array}{l}724 . \\
10\end{array}$ & $\begin{array}{l}98.4 \\
4 \\
\end{array}$ \\
\hline$S_{y}$ & 11.67 & $\begin{array}{l}56.457 \\
167\end{array}$ & $\begin{array}{l}8479.33 \\
801\end{array}$ & $\begin{array}{l}299 . \\
07\end{array}$ & 9.46 \\
\hline$S_{x}$ & $\begin{array}{l}23029 . \\
072\end{array}$ & $\begin{array}{l}40.674 \\
797\end{array}$ & $\begin{array}{l}6372.44 \\
072\end{array}$ & $\begin{array}{l}1607 \\
.57\end{array}$ & $\begin{array}{l}187 . \\
94\end{array}$ \\
\hline$\beta_{2}(y)$ & 16.523 & $\begin{array}{l}25.896 \\
9\end{array}$ & 40.8536 & $\begin{array}{l}24.1 \\
4\end{array}$ & $\begin{array}{l}27.9 \\
6\end{array}$ \\
\hline$\beta_{2}(x)$ & 17.516 & $\begin{array}{l}38.889 \\
8\end{array}$ & 48.1567 & $\begin{array}{l}26.1 \\
4\end{array}$ & $\begin{array}{l}28.1 \\
0\end{array}$ \\
\hline $\mathrm{h}$ & 14.398 & $\begin{array}{l}26.814 \\
2\end{array}$ & 43.7615 & $\begin{array}{l}20.8 \\
0\end{array}$ & $\begin{array}{l}23.0 \\
8\end{array}$ \\
\hline$\rho$ & 0.865 & 0.7213 & 0.9948 & 0.90 & 0.89 \\
\hline
\end{tabular}
per 100 tons

$\mathrm{X}$ : the auxiliary variable shows no of trees (1 unit shows 100 apple trees) 
Table 2 MSE of the Existing and the Proposed Estimators

\begin{tabular}{|c|c|c|c|c|c|c|}
\hline \multicolumn{2}{|c|}{ Estimators } & Data 1 & Data2 & Data3 & Data4 & Data5 \\
\hline Isaki (1983) & $t_{\text {Isaki }}$ & 4862.205231 & 3778793.028 & $1.92215 \mathrm{E}+14$ & 1827374379 & 39643.29503 \\
\hline \multirow{4}{*}{$\begin{array}{l}\text { Kadilar \& Cingi } \\
\text { (2005) }\end{array}$} & $t_{C H 1}$ & 4862.205422 & 3983111.977 & $1.92216 \mathrm{E}+14$ & 1827397122 & 39675.37353 \\
\hline & $t_{C H 2}$ & 4862.885344 & 4862.885344 & 4862.885344 & 4862.885344 & 4862.885344 \\
\hline & $t_{T C H 3}$ & 4862.205422 & 4862.205422 & 4862.205422 & 4862.205422 & 4862.205422 \\
\hline & $t_{C H 4}$ & 4862.885467 & 4862.885467 & 4862.885467 & 4862.885467 & 4862.885467 \\
\hline \multirow{5}{*}{$\begin{array}{c}\text { Subramani \& } \\
\text { Kumarapandiyan } \\
(2012 b)\end{array}$} & $t_{j g 1}$ & 4862.204945 & 3468925.171 & $1.92214 \mathrm{E}+14$ & 1827353716 & 39594.00714 \\
\hline & $t_{j g 2}$ & 4862.204372 & 3022246.855 & $1.92212 \mathrm{E}+14$ & 1827312394 & 39496.7733 \\
\hline & $t_{j g 3}$ & 4862.204659 & 3220379.202 & $1.92213 \mathrm{E}+14$ & 1827333054 & 39545.16751 \\
\hline & $t_{j g 4}$ & 4862.204945 & 3468925.171 & $1.92214 \mathrm{E}+14$ & 1827353716 & 39594.00714 \\
\hline & $t_{j g 5}$ & 4862.204659 & 3220379.202 & $1.92213 \mathrm{E}+14$ & 1827333054 & 39545.16751 \\
\hline $\begin{array}{l}\text { Proposed } \\
\text { Estimator }\end{array}$ & $t_{\text {new }}$ & 4857.818785 & 2846652.986 & $1.84037 \mathrm{E}+14$ & 1792753628 & 36249.92917 \\
\hline
\end{tabular}

Table 3: PRE of the Existing and the Proposed Estimators

\begin{tabular}{|c|c|c|c|c|c|c|}
\hline \multicolumn{2}{|l|}{ Estimators } & \multirow{2}{*}{$\begin{array}{l}\text { Data 1 } \\
4862.205231\end{array}$} & \multirow{2}{*}{$\begin{array}{l}\text { Data2 } \\
3778793.028\end{array}$} & \multirow{2}{*}{$\begin{array}{l}\text { Data3 } \\
1.92215 \mathrm{E}+14\end{array}$} & \multirow{2}{*}{$\begin{array}{l}\text { Data4 } \\
1827374379\end{array}$} & \multirow{2}{*}{$\frac{\text { Data5 }}{39643.29503}$} \\
\hline $\begin{array}{l}\text { Isaki } \\
(1983)\end{array}$ & $t_{\text {Isaki }}$ & & & & & \\
\hline \multirow{4}{*}{$\begin{array}{l}\text { Kadilar \& Cingi } \\
\text { (2005) }\end{array}$} & $t_{C H 1}$ & 4862.205422 & 3983111.977 & $1.92216 \mathrm{E}+14$ & 1827397122 & 39675.37353 \\
\hline & $t_{C H 2}$ & 4862.885344 & 4862.885344 & 4862.885344 & 4862.885344 & 4862.885344 \\
\hline & $t_{T C H 3}$ & 4862.205422 & 4862.205422 & 4862.205422 & 4862.205422 & 4862.205422 \\
\hline & $t_{C H 4}$ & 4862.885467 & 4862.885467 & 4862.885467 & 4862.885467 & 4862.885467 \\
\hline \multirow{5}{*}{$\begin{array}{l}\text { Subramani \& } \\
\text { Kumarapandiyan } \\
(2012 b)\end{array}$} & $t_{j g 1}$ & 4862.204945 & 3468925.171 & $1.92214 \mathrm{E}+14$ & 1827353716 & 39594.00714 \\
\hline & $t_{j g 2}$ & 4862.204372 & 3022246.855 & $1.92212 \mathrm{E}+14$ & 1827312394 & 39496.7733 \\
\hline & $t_{j g 3}$ & 4862.204659 & 3220379.202 & $1.92213 \mathrm{E}+14$ & 1827333054 & 39545.16751 \\
\hline & $t_{j g 4}$ & 4862.204945 & 3468925.171 & $1.92214 \mathrm{E}+14$ & 1827353716 & 39594.00714 \\
\hline & $t_{j g 5}$ & 4862.204659 & 3220379.202 & $1.92213 \mathrm{E}+14$ & 1827333054 & 39545.16751 \\
\hline Proposed Estimator & $t_{\text {new }}$ & 4857.818785 & 2846652.986 & $1.84037 \mathrm{E}+14$ & 1792753628 & 36249.92917 \\
\hline
\end{tabular}

\section{CONCLUSION}

In this article we have proposed a modified ratio-type variance estimator using known value of kurtosis of the auxiliary variable. The bias and mean square error of the proposed modified ratio-type variance estimator are obtained and compared with that of existing modified ratio type variance estimator and show that proposed estimator is more efficient than the existing estimator. We have also assessed the performances of the proposed estimator for known population.

\section{REFERENCES}

[1] Ahmed, M.S. and Hossain, M.I. (2000) . Some competitive estimators of finite Population variance Multivariate Auxiliary Information, Information and Management Sciences, Volume 11 (1), 49-54

[2] Arcos , C.A. and Rueda , G.M , variance estimation using auxiliary information an almost unbiased multivariate ratio estimator. Matrika , Vol.45, pp.171$178,1997$.
[3] Das, A.K, \& Tripathi, T.P. (1978). Use of auxiliary information in estimating the finite population variance . Sankhya , 40, 139-148 .

[4] Gupta , S , \& Shabbir, J. (2008). Variance estimation in simple random sampling using auxiliary information . Hacettepe Journal of Mathematics and Statistics, 37, 5767.

[5] Isaki , C.T . (1983) . Variance estimation using auxiliary information . Journal of the American Statistical Association, 78, 117-123.

[6] Kadilar, C , Cingi ,H. (2005) . A new ratio estimator in stratified sampling . Comm. Statist. Theory Meth. 34:1-6.

[7] Kadilar , C , Cingi ,H. (2006a). Improvement in variance estimation using auxiliary information. Hacettepe Journal of Mathematics and Statistics , 35(1), 111-115.

[8] Kadilar , C , Cingi ,H. (2006b) . Ratio estimators for population variance in simple and stratified sampling . Applied Mathematics and Computation , 173, 1047-1058 
International Journal of Computer Applications (0975 - 8887)

Volume 175 - No. 33, November 2020

[9] Subramani, J, \& Kumarapandiyan , G. (2012a). Estimation of population mean using coefficient of variation and median of an auxiliary variable. International Journal of Probability and Statistics , 1(4), 111-118.

[10] Subramani, J, \& Kumarapandiyan ， G. (2012b) . Variance Estimation using median of an auxiliary variable. International Journal of Probability and Statistics, 1(3) , 36-40.

[11] Subramani, J, \& Kumarapandiyan, G. (2013). Estimation of variance using known coefficient of variation and Median of an auxiliary variable. International Journal of Modern Applied Statistical Methods, 1(12) ,58-64. 